\title{
Observing nucleon decay in lead perchlorate
}

\author{
R. N. Boyd ${ }^{1,2}$ T. Rauscher, ${ }^{3}$ S. D. Reitzner, ${ }^{1}$ and P. Vogel ${ }^{4}$ \\ ${ }^{1}$ Department of Physics, Ohio State University, Columbus, Ohio 43210, USA \\ ${ }^{2}$ Department of Astronomy, Ohio State University, Columbus, Ohio 43210, USA \\ ${ }^{3}$ Department of Physics, University of Basel, CH-4056 Basel, Switzerland \\ ${ }^{4}$ Department of Physics, California Institute of Technology, Pasadena, California 91125, USA
}

(Received 21 July 2003; published 31 October 2003)

Lead perchlorate, part of the OMNIS supernova neutrino detector, contains two nuclei ${ }^{208} \mathrm{~Pb}$ and ${ }^{35} \mathrm{Cl}$ that might be used to study nucleon decay. Both would produce signatures that will make them especially useful for studying less-well-studied neutron decay modes, e.g., those in which only neutrinos are emitted.

DOI: 10.1103/PhysRevD.68.074014

PACS number(s): 13.30.Ce, 14.20.Dh

\section{INTRODUCTION}

Studies of nucleon decay (see, e.g., [1-5]) are among the most important in physics in that they provide direct tests of fundamental theories of particle physics. The different particle theories make rather different predictions as to what the nucleon decay half-lives might be, and even which decay modes would be expected to dominate. Although a number of searches for nucleon decay have been performed in the large detectors that exist, no convincing evidence has yet been presented for its existence. The best limits from these studies have involved decay modes in which protons decay to relativistic charged leptons (see, e.g., [6]), either as individual protons or as protons in nuclei, as the resulting Cherenkov radiation would produce definitive signatures, typically of order $10^{33} \mathrm{yr}$. However, nucleon decays in composite nuclei [7-10] might produce clear evidence for the existence of such effects that could not be obtained from the decay of isolated protons. Furthermore, these might allow the extension of the existing limits of the decay branches in some instances, even with relatively small detectors.

In this study we focus on the $n \rightarrow \nu+\bar{\nu}+\nu$ decay, which has a less-well-known half-life because its decay can often be masked by backgrounds, and would in any event be difficult to detect via the means used to observe nucleon decay into relativistic leptons. It is not necessarily expected to be the dominant mode of decay, but it is the least-welldetermined mode, and so it is the primary limitation to the mode-independent half-life. Moreover, there are nonstandard models in which unusual decay modes may turn out to be the dominant ones (see, e.g., [11]). In some models the three-neutrino decay mode can emerge naturally as the dominant one [12].

Decays of the three-neutrino mode were searched a decade ago, [13], then their limit was improved to its current experimental value of $5 \times 10^{26} \mathrm{yr}$ [6]. Recent suggestions for studying this decay mode have included signatures that would result from the decay of a neutron in the $\mathrm{O}$ in $\mathrm{H}_{2} \mathrm{O}$ [9] of Super-Kamiokande and in the $\mathrm{C}$ in the $\mathrm{CH}_{2}$ [10] of KamLAND. These suggestions both involve signals that would be generated by rather weak branches resulting from nucleon decay.

\section{SIGNALS FROM LEAD PERCHLORATE}

In this paper we study two nuclides ${ }^{208} \mathrm{~Pb}$ and ${ }^{35} \mathrm{Cl}$ which, we show, would produce special responses to neutron decay. We present the signatures that might result in the $n \rightarrow \nu \bar{\nu} \nu$ decay. We find that ${ }^{208} \mathrm{~Pb}$ would have good sensitivity to this mode, but ${ }^{35} \mathrm{Cl}$, while not producing as strong a limit, would exhibit an unusually definitive signature. Both nuclei are part of a planned detector, lead perchlorate (LPC) $\mathrm{Pb}\left[\mathrm{ClO}_{4}\right]_{2}$, that will be part of OMNIS, the Observatory for Multiflavor NeutrInos from Supernovae [14]. LPC is a colorless liquid that is highly soluble in water. The properties of such a mixed liquid have been studied extensively [15]; it was found that the LPC will produce Cherenkov light from relativistic particles, e.g., electrons and muons, that might be produced in many nucleon decays. In addition, each neutron produced by neutron emission from a nucleus within the detector will be captured on the ${ }^{35} \mathrm{Cl}$ within tens of microseconds, producing $8.6 \mathrm{MeV}$ of $\gamma$-rays. An energetic $\gamma$-ray will also produce Cherenkov light, with a pattern that is indistinguishable from that from the relativistic leptons in LPC.

In general we can write the following expression for the neutron decay lifetime $\tau_{n}$ :

$$
\tau_{n} / \mathrm{Br}>N_{n} \epsilon_{n} R_{d e t} \epsilon_{1} \epsilon_{2}^{n},
$$

where $N_{n}$ is the number of neutrons in, e.g., ${ }^{208} \mathrm{~Pb}$ or ${ }^{35} \mathrm{Cl}$, $\epsilon_{n}$ is the fraction of those that can decay into detectable signatures, $R_{d e t}$ is the observed event rate, and $\epsilon_{1}$ and $\epsilon_{2}$ are the detection efficiencies for the two (or more) signatures of the decay. The factor $\epsilon_{2}^{n}$ accounts for the possibility of multiple neutron emission from the decay of a neutron in ${ }^{208} \mathrm{~Pb}$, each with detection efficiency $\epsilon_{2}$. The factor $\mathrm{Br}$ is the branching ratio for the decays that go to the specific decay mode being studied. In the ${ }^{208} \mathrm{~Pb}$ decay, this could, e.g., refer to the branching ratio for producing one neutron and a subsequent $\gamma$-ray with sufficient energy to be observed; we have assumed $\mathrm{E}_{e x}<3 \mathrm{MeV}$ in the daughter nucleus for this criterion to be satisfied, as virtually every level with excitation energy above that will produce at least one $3-\mathrm{MeV} \gamma$-ray [16]. In the ${ }^{35} \mathrm{Cl}$ decay, $\mathrm{Br}$ refers to a decay to states that will produce a $\gamma$-ray with at least $3 \mathrm{MeV}$ of energy together with decay to the ${ }^{34} \mathrm{Cl}$ ground state. $\left({ }^{34} \mathrm{Cl}\right.$ has an isomeric state at 
$0.146 \mathrm{MeV}$, to which roughly half of the highly excited states will ultimately decay. However, it has a much longer half-life than the $1.5 \mathrm{~s}$ half-life of the ground state, so it will be assumed not to be useful for the present discussion.)

In order to estimate the probability of observing decays from ${ }^{208} \mathrm{~Pb}$ and ${ }^{35} \mathrm{Cl}$ we will have to consider the probability of population of sufficiently highly excited states in the daughter nuclei: ${ }^{207} \mathrm{~Pb}$ and ${ }^{34} \mathrm{Cl}$. Generally the more deeply bound the nucleon that decays, the larger will be the excitation energy in the daughter nuclide. The nuclide that decays will be a nuclide in the parent nucleus, so the resulting "state" of the initial nucleus, ${ }^{208} \mathrm{~Pb}$ or ${ }^{35} \mathrm{Cl}$, minus one neutron will map onto actual states in the daughter nuclide (assuming the neutron decay leaves the resulting nucleus intact; a reasonable assumption for the decay mode that produces three particles that interact only through the weak interaction). However, consider the energy $E_{\text {decay }}$ of the neutron decay products (e.g., the three neutrinos) when the final nucleus $\mathrm{A}-1$ is excited to the excitation energy $E_{\text {exc }}$. Then $E_{\text {decay }}=M_{n}-\left(S_{n}+E_{\text {exc }}\right)$, where $S_{n}$ is the neutron separation energy in the parent nucleus A. The energy in parentheses is just the binding energy of the neutron that decayed. Then, all other things being equal, extremely large values of $E_{\text {exc }}$ will be suppressed by the decrease of the phase space of the three neutrinos. This will tend to favor the excitation energies of the states in the daughter being similar to the binding energy of the neutron that decayed. Of course, this argument also assumes that the recoil kinetic energy of the residual nucleus will be negligible.

\section{A. The $n \rightarrow \nu \bar{\nu} \nu$ decay mode in ${ }^{208} \mathrm{~Pb}$}

As discussed above, the signature of this nucleon decay would be given by the specific properties of the residual nuclei resulting from the decay. In this case, ${ }^{208} \mathrm{~Pb}$ would first become ${ }^{207} \mathrm{~Pb}$. What would happen next would depend on the excitation energy of the states in ${ }^{207} \mathrm{~Pb}$ that were populated compared to the one-neutron emission threshold, the two-neutron emission threshold, etc., in ${ }^{207} \mathrm{~Pb}$. Subsequent neutron emissions will be much more rapid than electromagnetic deexcitations, so subsequent neutron emissions would occur instead of deexcitations of the daughter nuclei as long as the states populated were above the neutron-decay threshold. The excitation energy in the postdecay ${ }^{207} \mathrm{~Pb}$ will depend on the binding energy of the nucleon that decayed. This energy would be expected to be as much as several tens of $\mathrm{MeV}$ in lead, but would be weighted toward lower values by the preponderance of higher spin nuclear orbits, hence higher occupation numbers, near the Fermi surface. Thus a reasonable range to assume for the excitation energy in ${ }^{207} \mathrm{~Pb}$ might be an asymmetric distribution ranging from essentially zero (if a valence neutron decayed) to as much as $20 \mathrm{MeV}$, with an even higher-energy tail of the distribution extending to several more tens of $\mathrm{MeV}$, but with the highest energies suppressed. The one-neutron (two-neutron) emission threshold in ${ }^{207} \mathrm{~Pb}$ is $6.74 \mathrm{MeV}(14.83 \mathrm{MeV})$. Decays of neutrons in the highest-energy occupied shells would presumably primarily populate states below the one-neutron-emission threshold in ${ }^{207} \mathrm{~Pb}$. Although these states would decay by emitting $\gamma$-rays, ${ }^{207} \mathrm{~Pb}$ is stable. Thus the $\gamma$-rays would be the only signature of the neutron decays to the low-lying states, providing a less-than-compelling signature of nucleon decay. However, a fairly large fraction of the neutron decays in ${ }^{208} \mathrm{~Pb}$ would produce at least one neutron emission. These states would produce ${ }^{207-j} \mathrm{~Pb}$ plus $j$ neutrons, with $j$ being at least one.

The branchings into the particle and $\gamma$-ray emission channels at a given excitation energy of the daughter nucleus ${ }^{207} \mathrm{~Pb}$ have been calculated. The relevant transmission coefficients were determined with the same inputs used in the NON-SMOKER statistical model code $[17,18]$, which is often used for astrophysical calculations, and has been found to be accurate over a wide mass range $[19,20]$. In addition to single-particle emission, two-particle emission can be calculated, specifically for the case at hand, i.e., the two-neutron emission. For two-particle emission each transition was followed from a level with given spin and parity in ${ }^{207} \mathrm{~Pb}$ to a definite level with given spin and parity in ${ }^{206} \mathrm{~Pb}$, then probabilities for subsequent neutron emission were determined by summing over neutron emission to all possible final states in ${ }^{205} \mathrm{~Pb}$. Up to 20 low-lying experimentally known levels were used in each nucleus involved, and a theoretical level density [20] was employed above the last known state. The relative probability of one- and two-neutron emission as a function of excitation energy in ${ }^{207} \mathrm{~Pb}$ is shown in Fig. 1. Spins from $\frac{1}{2}$ to $\frac{29}{2}$ and both parities were considered in ${ }^{207} \mathrm{~Pb}$. The transitions to the states of different spin were weighted only with $2 J+1$. In order to obtain the correct decay probability, the calculated probabilities have to be folded with the function that describes how the ${ }^{207} \mathrm{~Pb}$ levels of a given excitation energy are populated in the primary decay event, as described above. The basic features of the present results will remain, although the actual distribution of strength would be expected to be distorted somewhat due to the folding with the population derived from the $\nu \bar{\nu} \nu$ decay.

As can be seen from Fig. 1, the neutron channel dominates the possible decays above the neutron emission threshold. Only up to about $9 \mathrm{MeV}$ is the decay governed by photon emission. Although the proton emission threshold is at $7.5 \mathrm{MeV}$, there is no significant proton emission up to 40 $\mathrm{MeV}$ in excitation due to the high Coulomb barrier; it is still only $20 \%$ of all decays at $48 \mathrm{MeV}$ of excitation energy. Although the two-neutron emission channel opens at 14.83 $\mathrm{MeV}$, it remains insignificant up to $30 \mathrm{MeV}$ in excitation energy reaching $40 \%$ of all transitions at $48 \mathrm{MeV}$. It should be noted that the solid curve in Fig. 1 contains the sum of one- and two-neutron emission. Therefore, emissions of single and double neutrons contribute equally at the highest calculated energy, each comprising $40 \%$ of all decay possibilities. Thus the population of excitation energies close to the neutron separation energy can be probed by measuring $\gamma$-rays, whereas higher excitation energies are accessible by two-neutron decay.

The lowest-lying 82 neutrons in ${ }^{208} \mathrm{~Pb}$ would be sufficiently strongly bound that their decay would be very likely to populate states at least above the one-neutron emission threshold. Some of the remaining 44 neutrons would also be 


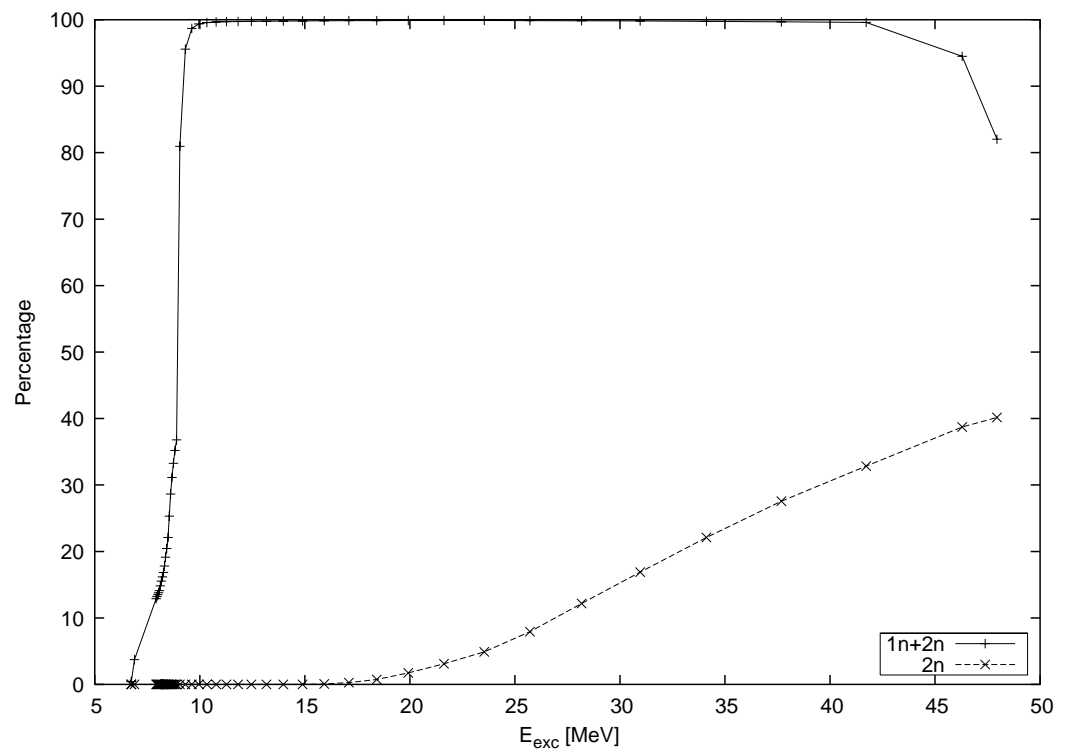

FIG. 1. The relative probability for one- and two-neutron emission as a function of excitation energy in ${ }^{207} \mathrm{~Pb}$. likely to produce daughter states above that threshold. Their ordering is $h_{9 / 2}, f_{7 / 2}, f_{5 / 2}, p_{3 / 2}, i_{13 / 2}$, and $p_{1 / 2}$, and their approximate binding energies are $12,11,8,8,8$, and $7 \mathrm{MeV}$, respectively [21]. It seems reasonable to assume that the decay of the neutrons in the last three orbitals would populate states below the one-neutron emission threshold. Thus 106 of the 126 neutrons in ${ }^{208} \mathrm{~Pb}$ would be expected to populate states above the one-neutron emission threshold in ${ }^{207} \mathrm{~Pb}$. An additional concern, though, is that the decays of neutrons in the most tightly bound states, i.e., those dominated by the $1 s_{1 / 2}, 1 p_{3 / 2}, 1 p_{1 / 2}, 1 d_{5 / 2}, 2 s_{1 / 2}$, and $1 d_{3 / 2}$ orbitals, might result in states in ${ }^{207} \mathrm{~Pb}$ that would decay by proton or $\alpha$-particle emission. Their binding energies are 40, 35, 35, 32, 31, and $29 \mathrm{MeV}$, respectively [21]. However, our emission probability calculations described above suggest that only the states having $1 s_{1 / 2}$ neutrons would be expected to exhibit such decays; we have subtracted the two neutrons in that orbital from consideration. Thus 104 neutrons in ${ }^{208} \mathrm{~Pb}$ can decay to produce detectable signatures.

The upshot is that this nucleon decay mode would have a high probability, roughly $104 / 126$ (so that $\epsilon_{n}$ [see Eq. (1)] is $104 / 126=0.825)$, for producing at least one neutron in coincidence with a fairly high-energy $\gamma$-ray, but emission of several neutrons in coincidence with a $\gamma$-ray would also have a relatively high probability. We have assumed $50 \%$ for the sum of the probabilities of events in which at least one neutron and a detectable $(>3 \mathrm{MeV}) \gamma$-ray are emitted. We note that states above $4 \mathrm{MeV}$ of excitation in ${ }^{207} \mathrm{~Pb}$ have a high probability, at least 50\% [16], of producing a $\gamma$-ray of at least $3 \mathrm{MeV}$ in energy.

We assume $1 \mathrm{kT}$ of lead perchlorate admixed with $20 \%$ water $\left(0.41 \mathrm{kT}\right.$ of lead) which has $5.9 \times 10^{29}{ }^{208} \mathrm{~Pb}$ nuclei or $6.1 \times 10^{31}$ neutrons in ${ }^{208} \mathrm{~Pb}$ that would be expected to populate states above the one-neutron emission threshold of ${ }^{207} \mathrm{~Pb}$ and decay subsequently by neutron emission. If the fraction of the 104 neutrons assumed that decay by single- or multiple- neutron emission is $100 \%$, the probability for de- tection of at least one neutron is $50 \%$ (it will be considerably higher for multiple-neutron emission), the probability for producing a detectable $\gamma$-ray is $50 \%$ and its detection efficiency is $50 \%$, then the probability that a neutron decay event from one of the 104 neutrons assumed to be detectable will be observed is $12.5 \%$. If the lifetime for this decay branch is $10^{30} \mathrm{yr}$, then, under these circumstances, seven to eight events per year would be observed. Note that we have assumed that only the ${ }^{208} \mathrm{~Pb}$ would contribute; it is likely that the other lead isotopes would produce similarly detectable decays, producing up to a factor of 2 enhancement. Furthermore, the assumed efficiencies are conservative. Thus this mode could have its lifetime extended by searching for these decays in lead, provided the background events could be managed.

The most obvious background signal that has several neutrons that would not be vetoed by a cosmic ray shield would involve production of those neutrons by an energetic cosmic ray in the surrounding rock. One might then get a $\gamma$-ray if one of the neutrons could inelastically excite a nucleus. However, this background could be eliminated easily, as the LPC detector could be surrounded by moderator, so that none of the neutrons getting into it would have enough energy to inelastically excite a nucleus.

A more serious background would result from neutrinos produced in the Earth's upper atmosphere inelastically exciting ${ }^{208} \mathrm{~Pb}$ via the neutral-current interaction to levels from which it could emit one or more neutrons, going to nuclei that might themselves emit more neutrons. This background would be impossible to reject on an event-by-event basis. However, its yield could be estimated as a function of the energy of the incident neutrino by measuring the number of charged-current interactions as a function of energy (as determined by the energy of the recoiling lepton) that would produce similar numbers of neutrons in coincidence with the $\gamma$-ray. One could then infer the yield from the neutral-current interactions on the basis of the relative magnitudes of the two types of cross sections and the energy distribution of the charged-current interactions. 


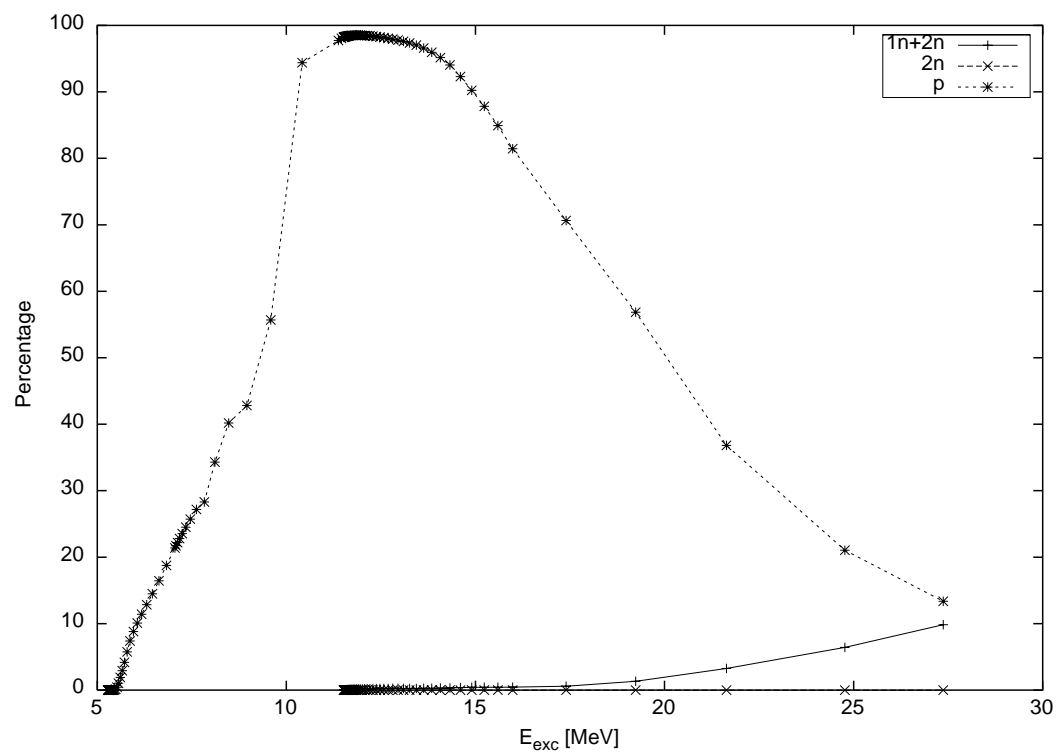

FIG. 2. The relative probability for one- and two-neutron emissions and one-proton emissions as a function of excitation energy in ${ }^{34} \mathrm{Cl}$.

\section{B. The $n \rightarrow \nu \bar{\nu} \nu$ decay mode in ${ }^{35} \mathrm{Cl}$}

The abundance of ${ }^{35} \mathrm{Cl}$ is $75 \%$ of natural $\mathrm{Cl}$, and there are $2 \mathrm{Cl}$ atoms per $\mathrm{Pb}$ atom in LPC. Thus it is also useful to see if the nucleon decay in ${ }^{35} \mathrm{Cl}$ might produce a definitive signature. Neutron decay in ${ }^{35} \mathrm{Cl}$ would sometimes produce ${ }^{34} \mathrm{Cl}$ in a highly excited state. However, the one-proton emission threshold for ${ }^{34} \mathrm{Cl}$ is at $5.14 \mathrm{MeV}$, far below the oneneutron emission threshold at $11.51 \mathrm{MeV}$. Indeed, there are apparently no bound levels in ${ }^{34} \mathrm{Cl}$ even close to $11.51 \mathrm{MeV}$. Thus the result of neutron decay to ${ }^{34} \mathrm{Cl}$ will be $\gamma$-ray decays to the ground state of ${ }^{34} \mathrm{Cl}$. Note, though, that ${ }^{34} \mathrm{Cl}$ has an isomeric state at $0.146 \mathrm{MeV}$; so roughly half of the energetic $\gamma$-rays would go to that state.

The relative branchings into different decay channels of ${ }^{34} \mathrm{Cl}$ were calculated using the same methods as described above for ${ }^{207} \mathrm{~Pb}$. The results are shown in Fig. 2. The situation for ${ }^{34} \mathrm{Cl}$ is different than for ${ }^{207} \mathrm{~Pb}$, however, because of the lower Coulomb barrier and a proton separation energy that is lower than the neutron separation energy. Neutron emission is relatively unimportant at all calculated excitation energies. The excited nucleus ${ }^{34} \mathrm{Cl}$ will deexcite via $\gamma$-transitions for excitation energies up to about $9.5 \mathrm{MeV}$, where proton emission accounts for $50 \%$ of all emissions. Proton emission quickly rises to nearly $100 \%$ by $11.9 \mathrm{MeV}$ and the falls off at high energies due to increased $\alpha$ emission. Two-neutron emission is completely negligible at all calculated energies. As with ${ }^{207} \mathrm{~Pb}$, a quantitative description of the emission requires the knowledge of the population of the excited states by the decay, but would not be expected to differ qualitatively from the results shown here.

The ground state of ${ }^{34} \mathrm{Cl}$, however, $\beta$-decays with a halflife of $1.53 \mathrm{~s}$. Thus a coincidence between the energetic $\gamma$-rays (assumed detection efficiency $=50 \%$ ) and the $\beta^{+}$-decay would identify a candidate event for neutron decay in ${ }^{35} \mathrm{Cl}$. The end point energy of the $\beta^{+}$is $4.47 \mathrm{MeV}$, so most of the $\beta^{+}$'s will be produced with sufficient energy to be detected from their Cherenkov radiation (assumed detection efficiency $=70 \%$ ). The isomeric state has a much longer half-life $(32 \mathrm{~m})$, so we have assumed that it would not produce a useful coincidence for determining nucleon decay (reducing the useful event yield by half). None the less, nucleon decay in ${ }^{35} \mathrm{Cl}$ could be identified by observing $\beta$ and $\gamma$ 's in coincidence, with enough events detected to confirm the half-life of ${ }^{34} \mathrm{Cl}$. The relatively long half-life of ${ }^{34} \mathrm{Cl}$ would demand that the materials used in OMNIS LPC modules be as pure as possible to minimize accidental coincidences.

Again assuming $1 \mathrm{kT}$ of lead perchlorate admixed with $20 \%$ water, there will be $1.8 \times 10^{30}{ }^{35} \mathrm{Cl}$ nuclei. Of the 18 neutrons in ${ }^{35} \mathrm{Cl}$, those in the $1 s_{1 / 2}, 1 p_{3 / 2}$, and $1 p_{1 / 2}$ orbits (binding energies $=36,27$, and $23 \mathrm{MeV}$, respectively [21]) would probably decay to sufficiently highly excited states in ${ }^{34} \mathrm{Cl}$, so that they would emit a proton, neutron, or $\alpha$-particle and not end up in ${ }^{34} \mathrm{Cl}$. The remaining three orbitals, $d_{5 / 2}$, $s_{1 / 2}$, and $d_{3 / 2}$ have binding energies of roughly 17,14 , and $10 \mathrm{MeV}$ [21]. The one-neutron separation energy in ${ }^{35} \mathrm{Cl}$ is 12.64 MeV; removal of a valence neutron, presumably from a $d_{3 / 2}$ dominated state, would require that much energy and would tend to populate the ground state of ${ }^{34} \mathrm{Cl}$. Thus, presumably, the decay of neutrons in ${ }^{35} \mathrm{Cl}$ states having strong $1 d_{5 / 2}$ or $2 s_{1 / 2}$ neutron configurations would tend to populate states in ${ }^{34} \mathrm{Cl}$ of sufficient excitation energy to emit a detectable, $>3 \mathrm{MeV}, \gamma$-ray. Thus, about 8 neutrons per ${ }^{35} \mathrm{Cl}$ nucleus, or $1.4 \times 10^{31}$ neutrons in the ${ }^{35} \mathrm{Cl}$ [the product $N_{n} \epsilon_{n}$ in Eq. (1)] would have appropriate binding energy to decay to states in ${ }^{34} \mathrm{Cl}$ that could produce a $\gamma$-ray above $3 \mathrm{MeV}$ in energy, so it would have a high probability of being detected. If the detection efficiency for the $\beta$ is assumed to be $70 \%$ and the lifetime for this decay process is $10^{30} \mathrm{yr}$, one would expect to observe three to four events per year from neutron decay in ${ }^{35} \mathrm{Cl}$. Although this decay mode would not produce as strong a limit on nucleon decay as would the lead, its signature would be considerably more definitive than that 
from the lead, because its backgrounds are so much more readily identifiable.

The primary background for the decay of a neutron in ${ }^{35} \mathrm{Cl}$ comes from neutrinos produced in the Earth's atmosphere, but such events would not be able to simulate nucleon decay events. Although once the neutron was knocked out of the ${ }^{35} \mathrm{Cl}$, the resulting $\gamma-\beta$ coincidence would be the same as for the neutron decay event, the atmospheric neutrino would emit a neutron, which would be detected to give its characteristic $8.6 \mathrm{MeV} \gamma$-ray. This is a considerably greater energy than could be produced by any of the $\gamma$-rays resulting from the deexcitations in ${ }^{34} \mathrm{Cl}$; thus that neutron could be used to veto this type of background event.

\section{Monte Carlo simulations of detectors to determine detection efficiencies}

The limit that can be achieved from any process, such as the decays described above, clearly depends on the detection efficiency. Thus we have run simulations of the events from decays of ${ }^{35} \mathrm{Cl}$ based on the GEANT [22] detector simulation software. As planned, the LPC detector will consist of multiple independent cylindrical modules having radii of $2 \mathrm{~m}$ and heights, which are adjustable in the simulations, ranging from 1 to $2 \mathrm{~m}$. The GEANT simulations were based on a single module. The interior of the cylinder was assumed to be viewed by photomultiplier tubes (PMTs) at both ends. Current design plans are to use an LPC solution of concentration, also adjustable in the simulations, of $75-80 \%$ LPC by weight.

From the decay of a neutron in ${ }^{35} \mathrm{Cl}$, GEANT generated both the $\gamma$-rays from the deexcitation of the ${ }^{34} \mathrm{Cl}$ nucleus and the resultant $\beta^{+}$from the decay of the ${ }^{34} \mathrm{Cl}$. The initial starting state for ${ }^{34} \mathrm{Cl}$ was randomly chosen from the energy levels that were below the proton separation energy. $\gamma$-rays were then generated to simulate the deexcitation of the ${ }^{34} \mathrm{Cl}$ nucleus from the selected starting state down to either the ground state or the isomer. The number and energies of the generated $\gamma$-rays were based on data on the levels and $\gamma$-rays for the ${ }^{34} \mathrm{Cl}$ nucleus. Events that decayed to the isomeric state were rejected. The fraction of the events that deexcite down to the ground state was found to be $57.6 \pm 0.2 \%$.

The ${ }^{34} \mathrm{Cl}$ decay time is chosen by randomly selecting whether a decay will occur during the 1-ms time unit, and was repeated until a decay occurred. The chance for a decay within a time unit was based on the half-life of ${ }^{34} \mathrm{Cl}$. If the decay time took longer than $40 \mathrm{~s}$, the total accumulated time was set to zero and the decay test continued.

The kinematics for the $\beta^{+}$were generated after the $\gamma$-rays were generated and tracked. The initial energy of the $\beta^{+}$was selected from a lookup table and the decay time was added to the initial time of flight. A Gaussian profile was used for the initial energy distribution of the positron. The parameters for the Gaussian were derived from a fit to the $\beta^{+}$energy spectrum calculated using the RADLST program [23].

Upon entering a PMT, a photon's energy and time of flight were stored. As $\beta^{+}$,s and $\beta^{-}$,s have a large RMS multiple scattering in LPC, any patterns in the Cherenkov radiation are destroyed. However, an event can be identified by the number of PMTs that have fired in a localized area within a specified time window. The requirement imposed to identify $\gamma$-rays and $\beta^{+}$, s required three PMTs to fire in a localized cluster within a 20 -ns window.

To estimate the efficiency for detecting neutron decay in the ${ }^{35} \mathrm{Cl}$ in LPC, 10000 events were generated in a $100-\mathrm{cm}-$ deep tank with an $80 \%$ solution of LPC by weight. The attenuation length of the LPC was taken to be $4.2 \mathrm{~m}$ [15]. The mean efficiency for detecting the $\gamma$-rays from the deexcitation of the ${ }^{34} \mathrm{Cl}$ nucleus over the volume of the detector was found to be $57.0 \pm 0.8 \%$. For detecting the $\beta^{+}$'s, the mean efficiency was found to be $75.2 \pm 0.9 \%$, making the mean efficiency for detecting a coincidence between the $\gamma$ 's and the $\beta$ 's to be $42.6 \pm 0.8 \%$. For a 200 -cm-deep tank with an $80 \%$ LPC solution, the mean detection efficiency for $\gamma$ 's, $\beta^{+}$'s, and coincidences was found to be $42.6 \pm 0.7 \%, 60.6$ $\pm 0.9 \%$, and $25.6 \pm 0.5 \%$, respectively. Thus the efficiencies assumed above are consistent with those determined from the Monte Carlo simulations. For the 200-cm-deep tank, the neutron detection efficiency was found to be $87.6 \pm 0.9 \%$.

The position of a nucleon decay event can be deduced from the time difference $\Delta t$ between hits at the two ends of the tank. $\Delta t$ can be defined in a variety of ways, e.g., by taking it to be the time difference between the peaks of the signals, either from the $\gamma^{\prime}$ 's or the $\beta^{+}$'s, from the two sides of the module, or by attempting to average early hits to utilize the leading edges of the signals on the two sides to do the timing. The first approach can be confused somewhat by the arrival of photons scattered from the opposite side, whereas the latter can be complicated by limited statistics. Either approach seems to allow localization of events to about $\pm 10 \mathrm{~cm}$. Spatial localization is important, as it allows use of a position dependent neutron detection efficiency, so it can allow greater emphasis on events that occur in the center of the detector, which will have a greater efficiency for detecting the veto neutron, than those at the edges, which will have a lesser efficiency.

\section{CONCLUSIONS}

Given that the suggested searches for nucleon decay would be conducted in a supernova neutrino detector, these searches would have a long time, probably more than $20 \mathrm{yr}$, to run. Thus the suggested experiments have the potential to improve greatly the existing limits on the $n \rightarrow \nu+\bar{\nu}+\nu$ decay mode.

\section{ACKNOWLEDGMENTS}

This work began at an Aspen Institute summer workshop. It has continued under the support of U.S. Department of Energy Grant No. DE-FG03-88ER40397, NSF Grant No. PHY-0099476, and the Swiss NSF (Grant No. 2000061031.02). T.R. acknowledges support by the Swiss NSF (Grant No. 2024-067428.02). 
[1] J.C. Pati and A. Salam, Phys. Rev. D 8, 1240 (1973); Phys. Rev. Lett. 31, 661 (1973); Phys. Rev. D 10, 275 (1974).

[2] H. Georgi and S.L. Glashow, Phys. Rev. Lett. 32, 438 (1974).

[3] K.S. Babu, J.C. Pati, and F. Wilczek, Nucl. Phys. B566, 33 (2000).

[4] K.S. Babu and R.N. Mohapatra, Phys. Lett. B 518, 269 (2001).

[5] T. Appelquist, B.A. Dobrescu, E. Ponton, and H.-U. Yee, Phys. Rev. Lett. 87, 181802 (2001).

[6] Particle Data Group, H. Hagiwara et al., Phys. Rev. D 66, 010001 (2002); http://pdg.lbl.gov

[7] Y. Totsuka, in Proceedings of the 7th Workshop on Grand Unification/ICOBAN 1986, edited by J. Arafune (World Scientific, Singapore, 1986), p. 118

[8] H. Ejiri, Phys. Rev. C 48, 1442 (1993).

[9] Y. Suzuki et al., Phys. Lett. B 311, 357 (1993).

[10] Y. Kamyshkov and E. Kolbe, Phys. Rev. D 67, 076007 (2003).

[11] J. Pati, A. Salam, and U. Sarker, Phys. Lett. 133B, 330 (1983).

[12] R.N. Mohapatra and A. Perez-Lorenzana, Phys. Rev. D 67, 075015 (2003).

[13] C. Berger et al., Phys. Lett. B 269, 227 (1991).
[14] R.N. Boyd, A.StJ. Murphy, and R.L. Talaga, Nucl. Phys. A718, 222c (2003).

[15] S.R. Elliott, Phys. Rev. C 62, 065802 (2000).

[16] Evaluated Nuclear Structure Data File, http:// www.nndc.bnl.gov/nndc/ensdf

[17] T. Rauscher and F.-K. Thielemann, At. Data Nucl. Data Tables 75, 1 (2000)

[18] T. Rauscher and F.-K. Thielemann, At. Data Nucl. Data Tables 79, 47 (2001)

[19] E. Kolbe, K. Langanke, S. Krewald, and F.-K. Thielemann, Nucl. Phys. A540, 599 (1992)

[20] T. Rauscher, F.-K. Thielemann, and K.-L. Kratz, Phys. Rev. C 56, 1613 (1997)

[21] A. Bohr and B.R. Mottelson, Nuclear Structure (World Scientific, Singapore, 1998), p. 239.

[22] GEANT 3.21, CERN Program Library, Long Writeup W5013 (1993).

[23] T.W. Burrows, computer code RADLST, Brookhaven National Laboratory Report No. BNL-NCS-52142, 1988. 